\title{
MANAGEMENT OF HYPERPROLACTINEMIA
}

Minie Mohan

1. Associate Professor. Department of Obstetrics \& Gynaecology, Katihar Medical College,

\section{CORRESPONDING AUTHOR:}

Minie Mohan, 601, Bimla Raj Enclave, Near Nalanda Medical College, Kankarbagh, Patna-20, Bihar, E-mail: minianand@yahoo.co.in Ph: 00919343111440

ABSTRACT: Hyperprolactenemia refers to the consistent presence of abnormally high levels of prolactin in the blood after ruling out the physiological causes of prolactin hypersecretion. Prolactin is mainly concerned with breast development during pregnancy and induction of lactation. It is secreted in a pulsatile manner, and increases with sleep, stress, pregnancy, chest wall stimulation or trauma. Therefore, hyperprolactenemia could be physiological, pathological or pharmacological. The clinical presentation is varied and may manifest as oligomenorrhoea, amenorrhoea, infertility (as a result of the above), decreased libido and habitual abortion. Premenopausal women may also present with galactorrhoea which requires the presence of both oestrogen and prolactin. Hyperprolactenemia may cause osteopenia and also hyperandrogenism Before giving treatment due consideration must be given to an infertile women regarding her desire for pregnancy. There are three treatment options :- Medical, Surgical and Radiotherapy. All have their pros and cons but medical treatment should be started as soon as possible. It also helps at the time of surgery as it may induce tumor regression enabling resection of a greater part of the tumor leading to better control of prolactin levels.

\section{INTRODUCTION:}

- Hyperprolactinemia is the most common endocrine disorder of the hypothalamicpituitary axis.

- It refers to the consistent presence of abnormally high levels of prolactin in the blood after ruling out the physiological causes of prolactin hypersecretion. Normal levels are less than $580 \mathrm{mlU} / \mathrm{L}$ for women and less than $450 \mathrm{ml} \mathrm{U} / \mathrm{L}$ for men.

- Prolactin is a 198-amino acid protein (23-kD) produced in the lactotroph cells of the anterior pituitary gland and its principal action is production and spontaneous flow of breast milk.

- Prolactin Secretion

- Prolactin secretion is regulated by the predominantly tonic inhibitory effect of the hypothalamus.

- The (4) hypothalamus secretes a prolactin-release-inhibiting factor (PIF) and a prolactin-releasing factor (PRF). 


\section{REVIEW ARTICLE}

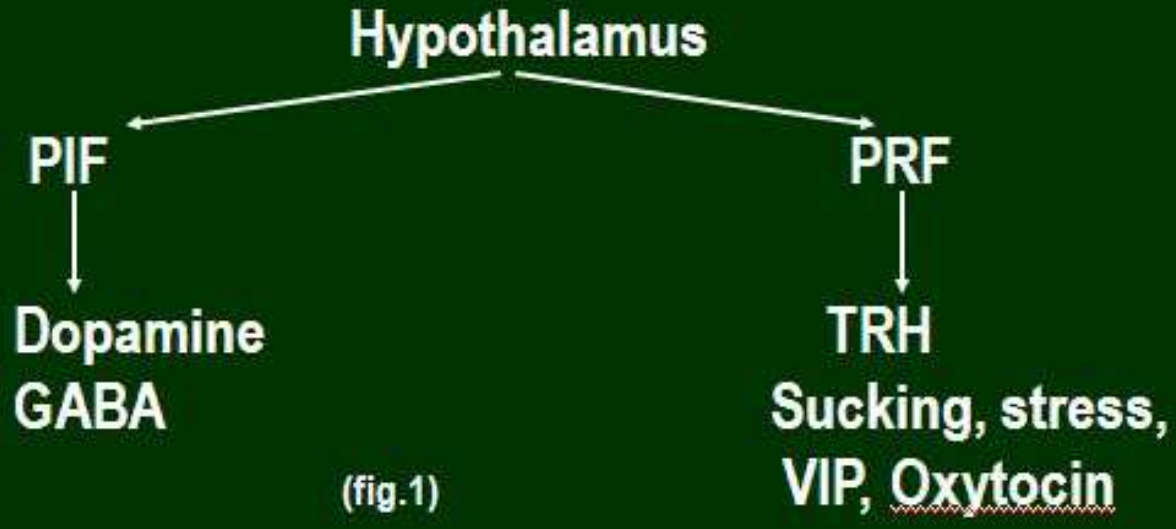

Pathophysiology of Hyperprolactinemia

The prolactin inhibitory factors reach the pituitary via the hypothalamic-pituitary portal vessels.

\section{Hypothalamus (PIF-Dopamine)}

\section{Hypothalamic - pituitary}

portal vessels

Anterior pituitary (D2 receptors)

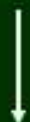

\section{Blockade of prolactin secretion}

\section{(fig.2)}

\section{CAUSES OF HYPERPROLACTINEMIA(5)}

\section{Physiological Causes:}

- Pregnancy

- Nursing

- Nipple stimulation

- Sexual Intercourse

- Stress (Surgery, hypoglycemia, myocardial infarction, syncope, trauma, venesection)

- $\quad$ Sleep

- Exercise

- Food Ingestion 


\section{CAUSES OF HYPERPROLACTINEMIA}

\section{Pharmacological causes:}

- Dopamine receptor antagonists

- Antipsychotics - Phenothiazines, Butyrophenones, Thioxanthesis, Resperidone, Sulpiride

- Antiemetics - Metoclopramide, Domperidone

- Dopamine - depleting agents -- a-Methyldopa, Reserpine

- Antidepressants, Tricyclic antidepressants

- Selective serotonin receptor Inhibitors

- Hormones -- Estrogens (high dose), Antiandrogens

- Opiates

- Verapamil

- Cimetidine (intravenous)

\section{CAUSES OF HYPERPROLACTINEMIA}

\section{Pathological causes:}

- Pituitary disease

- Prolactionomas

- $\quad$ Mixed GH/PRL - or ACTH/PRL - secreting adenomas

- Intrasellar tumors causing stalk compression (non secretory adenomas, germinoma, meningioma, glioma, metastasis)

- Intrasellar cyst

- Hypothalamic and pituitary stalk disease

- Granulomatous disease (sarcoidosis, tuberculosis, eosinophilic granuloma)

- Tumors (craniopharyngioma, harmartoma, glioma, germinoma, metastasis)

\section{Clinical presentation :}

Hyperprolactinemia may manifest as

Oligomenorrhoea,

Amenorrhea,

Infertility,

Decreased libido

Habitual abortion.

Osteopenia may occur due to prolonged hypoestrogenism induced by Hyperprolactinemia (4). 


\section{REVIEW ARTICLE}

Clinical presentation : It adversely affects fertility, potentially impairing GnRH pulsatality and thereby ovarian function

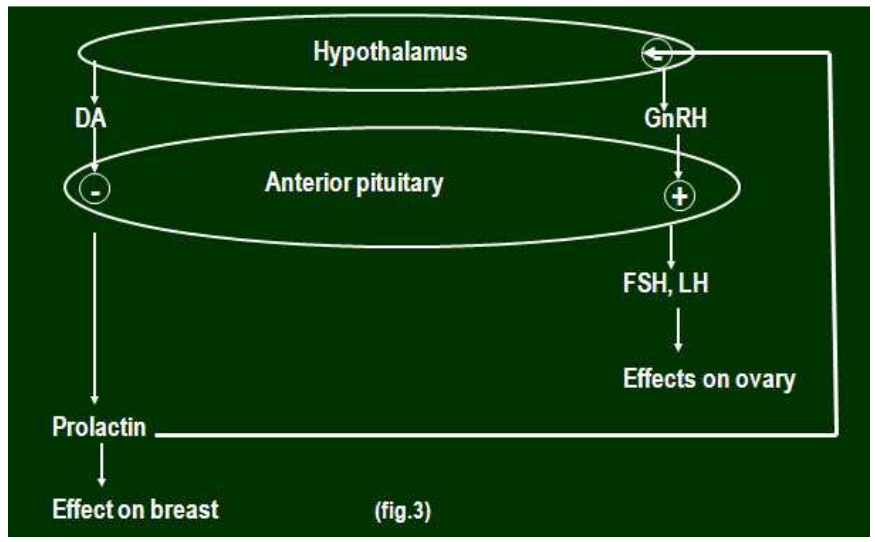

Hyperprolactinemia has a strong correlation with hypothyroidism in infertile women. (1)

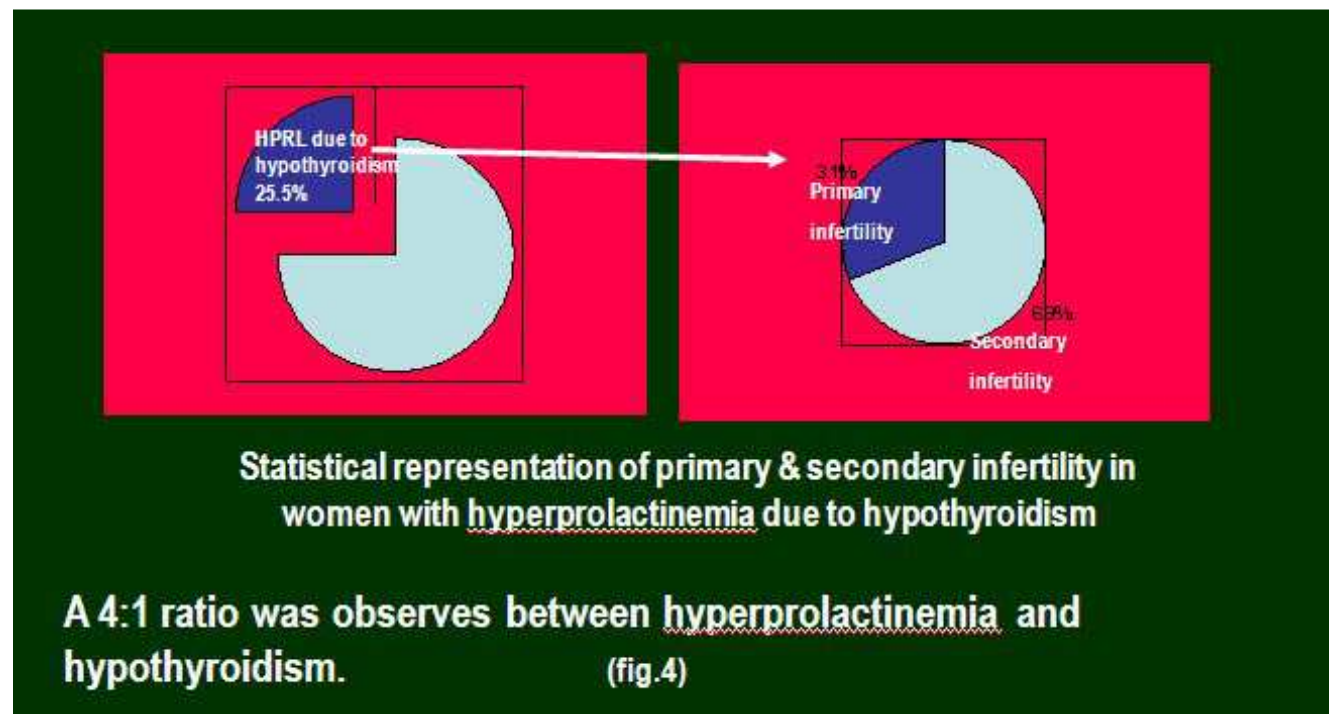

Also in a subset of women with PCOS mild to moderate Hyperprolactinemia is frequently detected.(1) These findings have suggested that some women with PCOS may have a disruption of the neuroendocrine mechanism particularly a deficiency of hypothalamic dopamine which is normally responsible for the release of gonadotropins and prolactin.

Hyperprolactinemic women may also have signs of chronic hyperandrogenism,(7) such as hirsutism and acne, possibly due to increased dehydroepiandrosterone sulfate secretion from the adrenals.

Treatment: Before starting treatment, due consideration must be given to several aspects, such as the patient's clinical presentation, the differences between microadenomas and microadenomas concerning their natural history, the desire for pregnancy, and the patient's treatment preference. Hyperprolactinemia can be treated by medications, surgery or radiotherapy. 
Medical Treatment: The current gold standard medical treatment of Hyperprolactinemia is with dopamine agonist drugs, such as bromocriptine, Cabergoline and quinagolide.(1)

Cabergoline has a long half-life, allowing once or twice weekly dosing and a better tolerability profile. However, data on its safety in pregnancy is limited.

Bromocriptine is a time tested drug but has side effects like dizziness, nausea, postural hypotension and GI symptoms.

There is also adequate data that it can be safely used in pregnancy as there is no evidence of teratogenecity in animal studies and in 1400 women taking BC when they conceived there was no evidence of increased incidence of abortion, multiple preg or fetal abnormalities.

However the possibility of unexpected late effect can not be excluded. In order to minimise foetal exposure to $\mathrm{BC}$ it is suggested that pts should initially use mechanical contraception.

Once regular menstrual cycle résumés contraceptive precaution is withdrawn so that pregnancy can be suspected as soon as menstrual period is $48 \mathrm{hrs}$ overdue and after confirming pregnancy by a serum BHCG assay and confirm pregnancy BC can be stopped

A randomized controlled trial was carried out to compare the effect of $\mathrm{BC}$ vs $\mathrm{CAB}$ on pregnancy in hyperprplactenemic infertile women.(3)

183 women with Hyperprolactinemia with 4.5 yrs of infertility were taken into the trial. Pts divided in 2 groups.

Group A -- containing 94 women treated with bromocriptine

Group B -- had 89 women treated with cabergolin.

For all women hormonal assay test (TSH, FSH, LH, prolactin) were carried out.

High rate of pregnancy and normal range of prolactin was observed in cabergolin group than compared to bromocriptine group with fewer side effects at the end of the study.

As experience with cabergolin has accumulated it appears that some pts who could not be controlled with other dopamine agonists eg (fig.5) bromocriptine or pergolide could be controlled by cabergolin

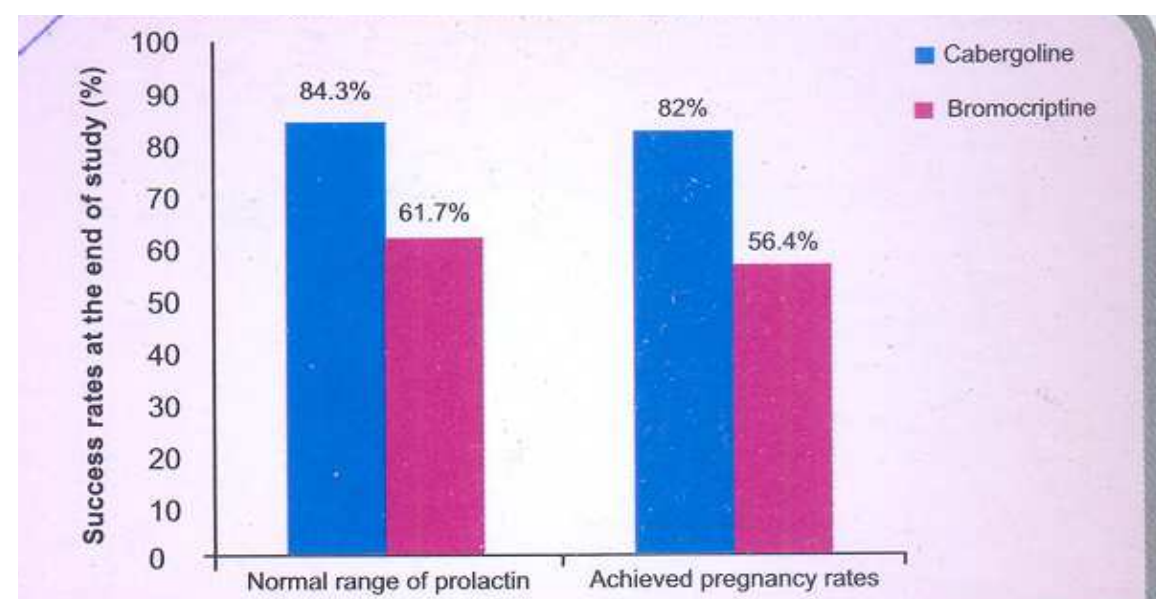

(fig.5)

Estrogen therapy may be used to replace dopamine agonists in women with idiopathic Hyperprolactinemia (IH) or microprolactinoma who do not want to become pregnant and those 
suffering from estrogen deficiency, particularly if they are resistant to or intolerant of dopamine agonists.

Surgical Treatment: In patient requiring surgical resection of prolactinomas, preoperative treatment with dopamine agonists can favorably alter the outcome of surgical intervention.

This was the finding of a study which evaluated 253 patients with prolactinomas treated surgically. Both short - and long-term outcomes of patients treated with dopamine agonists prior to surgery were compared with those undergoing surgery as their initial treatment modality.(2)

The results showed that patients treated with dopamine agonists prior to surgery experienced greater reductions in prolactin levels, had lower prolactin levels, were more likely to have normal prolactin levels at long term follow-up, and were less likely to require additional therapy to control their prolactin levels.

These results provide strong evidence that, regardless of initial prolactin level, pretreatment with dopamine agonist drugs may induce tumor regression, enabling resection of a greater percentage of the tumor and leading to better control of the prolactin level.

Pituitary surgery, usually by the transsphenoidal approach, is generally reserved for prolactinomas resistant to dopamine agonist drugs.

Transsphenoidal surgery offers the possibility of achieving complete cure in selected patients, although at the expense of significant risk in terms of morbidity, especially hypopituitarism, and a very low mortality rate. (1)

Microadenomas are more amenable to surgery, with normal levels of serum prolactin being achieved in about $80 \%$ cases; but the recurrence rate is also high at $\sim 25 \%$ at 5 years. Surgical results in macroprolactinomas are much poorer, mainly in big and/or invasive tumors.

Radiotherapy: Fractionated radiotherapy is now rarely used in primary therapy of prolactinomas because of relatively poor results and also because it is fraught with the risk of post radiotherapy pituitary insufficiency. Gamma knife radiosurgery (GKS), in which radiation to the pituitary area is more focused, offers potential advantages over conventional forms of radiotherapy, as it can diminish the radiation dose to normal tissues.(7) (1)

Conclusion: Hyperprolactinemia refers to the consistent presence of abnormally high levels of prolactin in the blood after ruling out the physiological causes of prolactin hypersecretion.

The commonest causes of chronic hyperprolactinemia are a prolactinoma after pregnancy, primary hypothyroidism, and drugs that elevate serum prolactin levels.

Treatment should aim to normalize prolactin levels, restore gonadal function, and reduce the effects of chronic hyperprolactinemia.

Clinical signs and symptoms of hyperprolactinemia are usually resolved when prolactin levels are lowered to within the normal range.

Dopamine agonists are the treatment of choice for the majority of patients.

Transsphenoidal surgery is resaved for pts who are intolerant to or resistant to dopamine agonists or when hyperprolactinemia is caused by non-prolactin-secreting tumors compressing the pituitary stalk. 


\section{REFERENCES:}

1. Melmed S, Casanueva FF, Hoffman AR, et al. Diagnosis and treatment of hyperprolactinemia: an endocrine society clinical practice guideline. J Clin Endocrinol Metab. Feb 2011;96(2):273-88.

2. Kharlip J, Salvatori R, Yenokyan G, Wand GS. Recurrence of hyperprolactinemia after withdrawal of long-term cabergoline therapy. J Clin Endocrinol Metab. Jul 2009;94(7):2428-36

3. Dekkers OM, Lagro J, Burman P, et al. Recurrence of hyperprolactinemia after withdrawal of dopamine agonists: systematic review and meta-analysis. J Clin Endocrinol Metab. Jan 2010;95(1):43-51

4. Biller MKB, Daniels GH. Neuroendocrine regulation and diseases of the anterior pituitary and hypothalamus. In: Braunwald E, Isselbacher KJ, Wilson J, et al. Harrison's Principles of Internal Medicine. 14th ed. New York, NY: McGraw-Hill; 1998:1974-8

5. Blackwell RE. Hyperprolactinemia. Evaluation and management. Endocrinol Metab Clin North Am. Mar 1992;21(1):105-24

6. Conner P, Fried G. Hyperprolactinemia; etiology, diagnosis and treatment alternatives. Acta Obstet Gynecol Scand. Mar 1998;77(3):249-62

7. Jones TH. The management of hyperprolactinaemia. Br J Hosp Med. Apr 19-May 2 1995;53(8):374-8. 